\title{
Wide diameter implants for single tooth restorations in the molar region: A retrospective study
}

IMPLANT THERAPY OUTCOMES, PERI-IMPLANT BIOLOGY ASPECTS
Jérémy Abitbol ${ }^{1}$ and Hadi Antoun ${ }^{1}$

Institut de Formation en Chirurgie Implantaire Avancée (IFCIA), Paris, France

\section{Background}

mmediate placement and loading of dental implants in fresh extraction sites are demanding protocols for achieving high primary stability and low bone resorption. These challenges are particularly important in the molar region. ${ }^{2,3}$ To meet the needs of patients requiring tooth replacement in such indications, wide diameter variable-thread apered implants were developed (Figure 1).

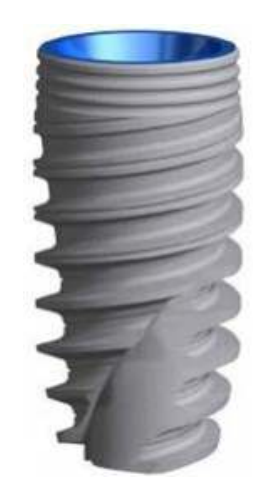

Figure 1 NobelActive WP $(5.5 \mathrm{~mm})$ implant used in this study for replacement of teeth in the molar region.

\section{Aim}

This retrospective study evaluated the safety and efficacy of wide diameter variablethread tapered implants placed primarily in fresh extraction sites in the molar region.

\section{Methods and Materials}

Consecutive patients were included who received at least one wide diameter variable-thread tapered implant (NobelActive WP, Nobel Biocare AB, Göteborg Sweden) in the molar region between April and June 2014

Patients with bone defects were excluded.

Grafting was performed in all fresh extraction sites using anorganic bovine bone matrix. No covering membranes were used.

A provisional restoration was placed immediately when the insertion torque was at least $35 \mathrm{Ncm}$ (range $35-70 \mathrm{Ncm}, \mathrm{n}=9$ ).

Implant stability was assessed by rocking and tapping

Radiographs were taken as part of routine treatment. Marginal bone remodeling was calculated from paired radiographs from baseline (implant insertion) and the last follow-up visit (mean 10.7 \pm 2.0 months, range $7-13$ months)

Descriptive statistical analysis was performed using SPSS 24 (IBM, USA).

\section{Results}

10 wide diameter $(5.5 \mathrm{~mm})$ variablethread tapered implants (length 7-13 $\mathrm{mm}$ ) were placed in the mandibular molar region of 9 patients (6 female, 3 male).

- The mean age of patients was 63.1 years (range $34-85$ years)

9 implants were place in the septum of fresh extraction sites and 1 was placed in a previously healed site.

9 implants were immediately loaded (<48 h). 1 implant, with an insertion torque of $20 \mathrm{Ncm}$, was fitted with a healing abutment and loaded with the final prosthesis after a healing period of 6 months.

- Mean marginal bone remodeling was $-0.32 \pm 1.42 \mathrm{~mm}$ (Table 1)

All implants survived and were stable at last follow-up.

\section{Clinical Case I}

A 61 year-old female patient presented with a periodontal lesion in the molar region (position 36). The infected tooth was extracted and replaced with a $5.5 \times 13 \mathrm{~mm}$ NobelActive WP implant in the fresh extraction socket. The implant was immediately loaded with a temporary crown. A cement-retained definitive restoration was placed 9 months later.

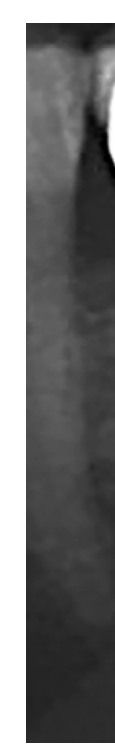

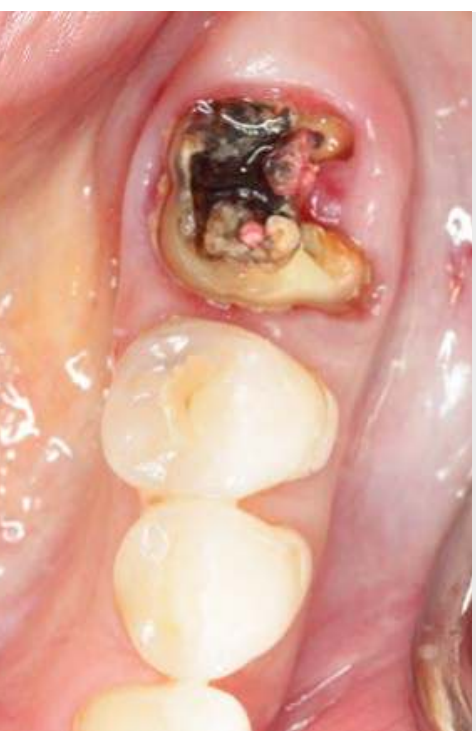

Figure 2 Radiograph (left) and clinical image (right) of molar (position 36) prior to extraction due to a periodontal lesion.

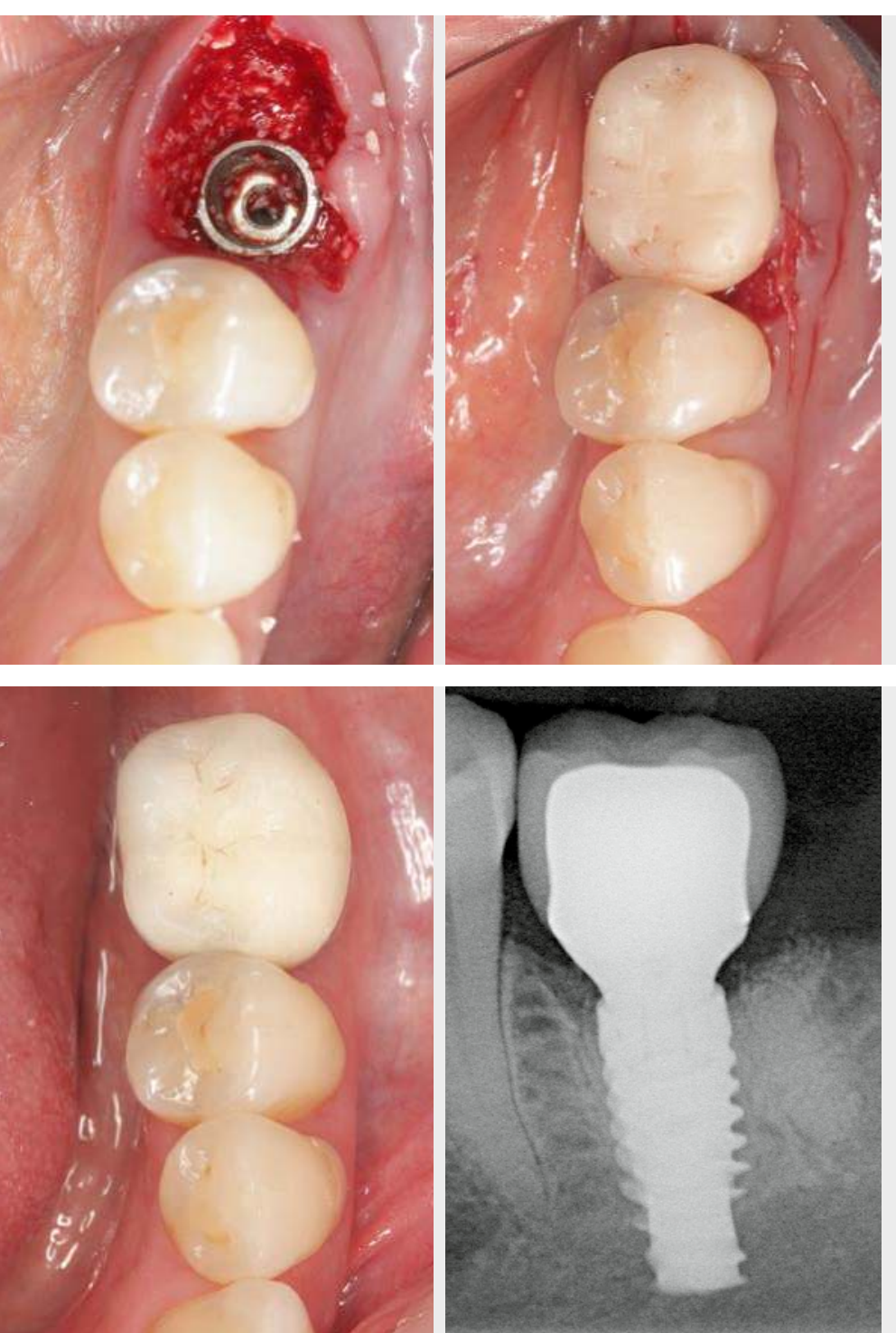

Figure 3 Insertion of $5.5 \times 13 \mathrm{~mm}$ implant the fresh extraction socket (left) and 作 visional crown (right).

\section{Clinical case II}

A 78 year-old male patient presented with a periodontal lesion at tooth 36 . The tooth was extracted in two stages and a $5.5 \times 8.5 \mathrm{~mm}$ NobelActive WP implant was inserted into the fresh extraction socket. The implant was immediately loaded with a provisional restoration. The definitive crown was placed 10 months after intervention. At final follow-up (10 months), the patient showed excellent marginal bone levels.

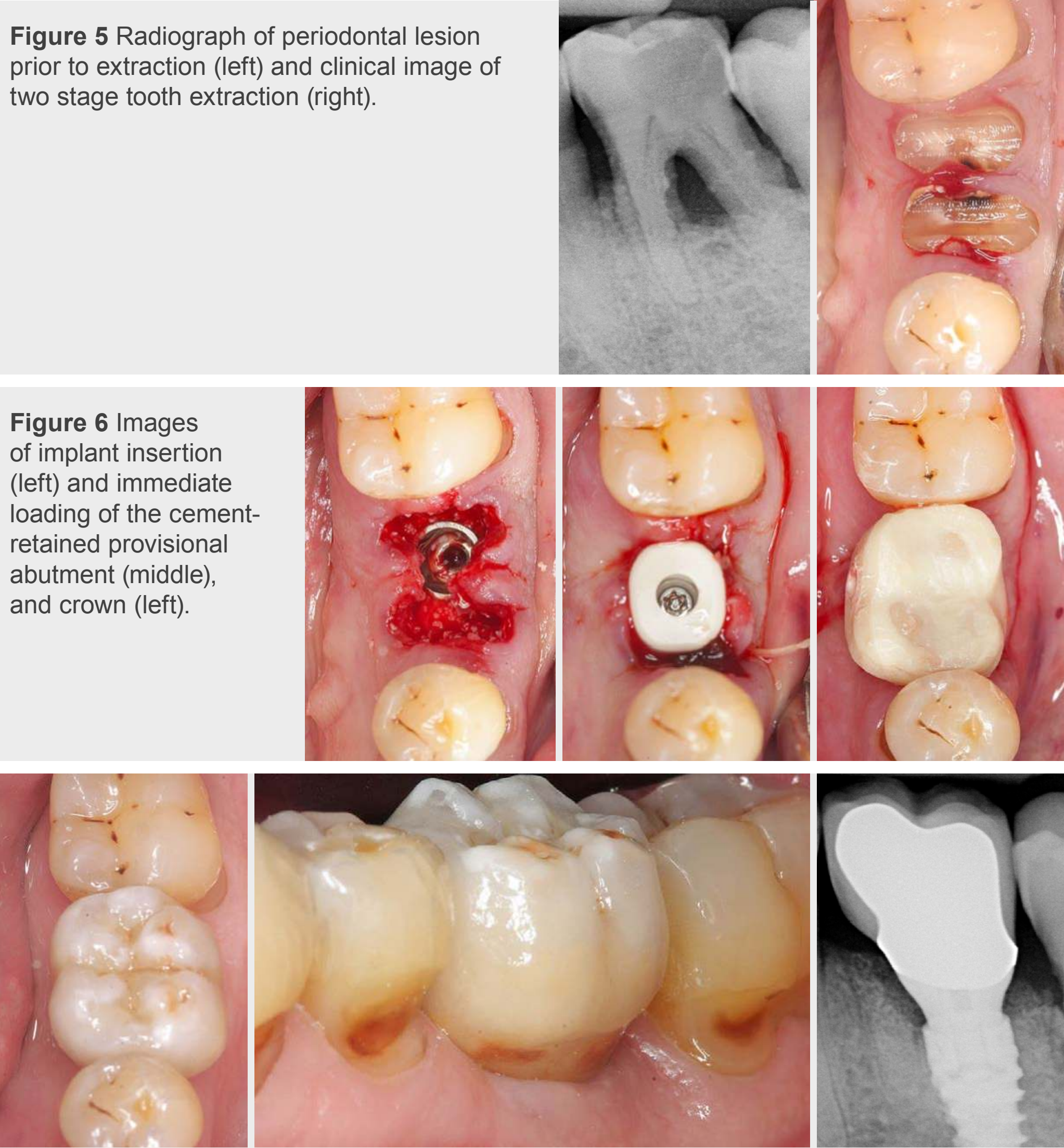

Figure 7 Images of occlusal (left) and clinical (middle) views of the definitive restoration

(10 months after implant placement). Radiograph (right) of definitive restoration at last follow-up.

\section{Conclusions}

The favorable results observed in this study suggest that wide diameter variablethread tapered implants are a safe and viable option for indications requiring the placement and immediate loading of implants in molar extraction sites.

\section{References}

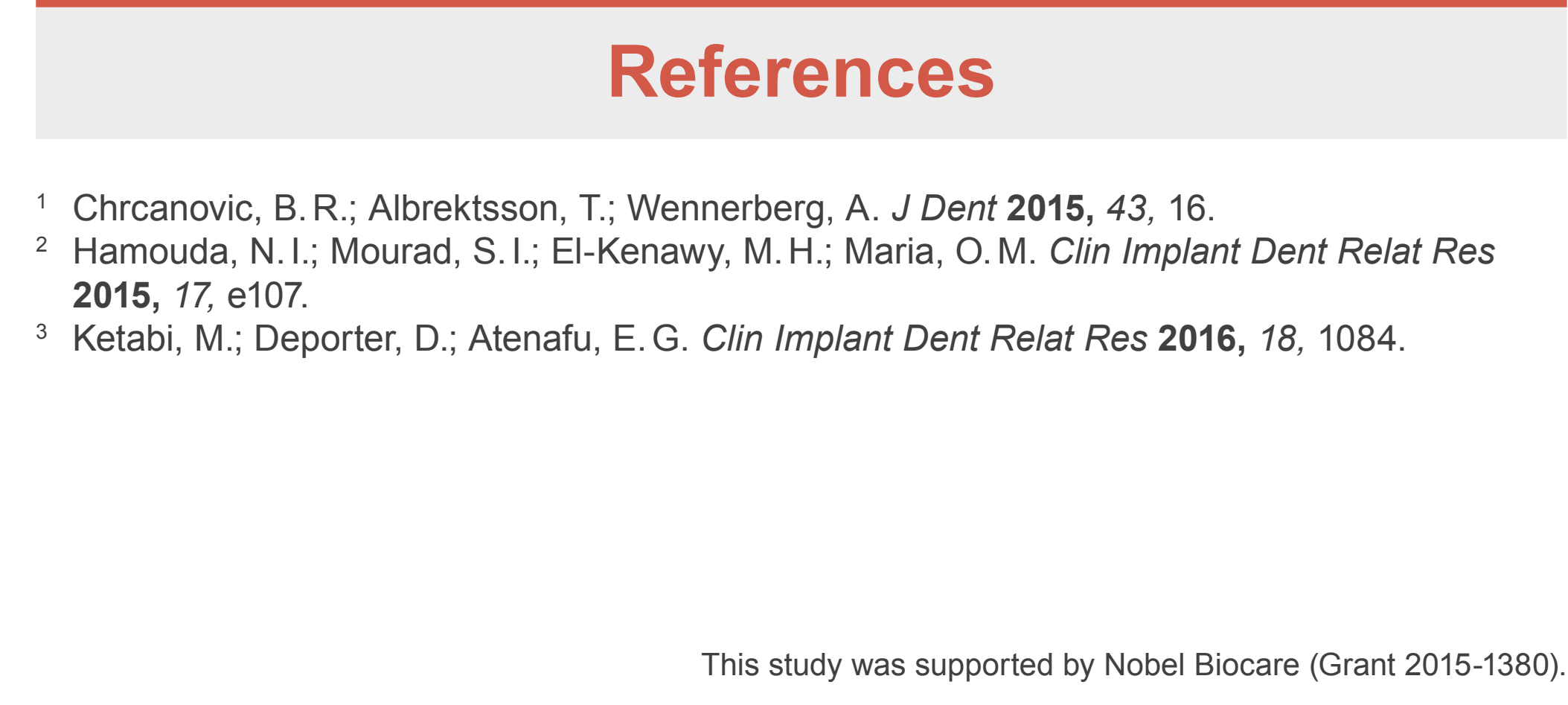

